Esta sección esta abierta para todos los lectores de la Revista, a la que pueden enviar sus preguntas, comentarios o críticas a los artículos que hayan sido publicados en los últimos números, teniendo en cuenta la posibilidad de que los autores aludidos puedan responder. Podrá aceptarse la comunicación de investigaciones preliminares, o de intervenciones en brotes que no hayan sido publicados ni sometidos a publicación en otra revista; así como, algunos comentarios sobre problemas de salud pública, ética y educación médica. La extensión máxima aceptable es de 1000 palabras, con un máximo de seis referencias bibliográficas (incluyendo la referencia del artículo que la motivó, cuando sea el caso) y una tabla o figura. Esta puede ser enviada a revmedex@ins.gob.pe.

\section{TRADUCCIÓN DE LA INVESTIGACIÓN EN ACCIONES ESPECÍFICAS EN SALUD}

\section{[TRANSLATION OF RESEARCH RESULTS IN SPECIFIC HEALTH ACTIONS]}

Fabián Fiestas ${ }^{1}$

Sr. Editor. En octubre de 2009, durante la entrega de Premios en Ciencia, Tecnología e Innovación en la Casa Blanca, el presidente de los Estados Unidos, Barack Obama, especificó: En tiempos difíciles como éste, hay quienes dicen que no debiéramos invertir en ciencia, que es un lujo hacerlo en un momento definido por las necesidades. Luego continuó: Yo no puedo estar más en desacuerdo con eso. La ciencia nunca ha sido más esencial como lo es ahora [de momentos difíciles] para nuestra prosperidad, nuestra seguridad, nuestra salud, y para nuestro modo de vida (1). Evidentemente, esta visión del Presidente norteamericano puede extenderse al contexto de los países pobres, como el Perú, donde los recursos han sido históricamente escasos y las necesidades crónicamente grandes. El enfoque es que cuanto más escasos son los recursos, mayor necesidad hay de gastarlos con sabiduría, y la ciencia aplicada nos ayuda a proceder así.

Deun manejo inteligente de los pocos recursos que tenemos en salud es de lo que trata la propuesta que aquí planteo, en concordancia con la corriente mundial que recomienda el uso de la evidencia científica para asegurar la efectividad de los gastos públicos en salud (2). Esta propuesta consiste en la creación estructural y funcional de un Centro (o Área), dentro del Ministerio de Salud o del Instituto Nacional de Salud, que tenga la misión exclusiva y especializada de traducir la información científica en información técnica lista a ser convertida en políticas, programas o intervenciones. Este Centro no tendría la función de generar investigación,

\footnotetext{
1 Médico Epidemiólogo. Laboratorios de Investigación y Desarrollo Facultad de Ciencias y Filosofía, Universidad Peruana Cayetano Heredia, Lima, Perú.
}

Recibido: 26-08-10 Aprobado: 01-09-10 sino de buscar activamente información que haya derivado de investigaciones locales o internacionales, para luego analizar y contrastar esta información con nuestra realidad, permitiéndonos tener propuestas de acción que sean contextualizadas y específicas a las necesidades y circunstancias locales.

Una iniciativa como esta produciría de manera continua y sostenida propuestas técnicas que ayudarán a los actores con responsabilidades políticas y de decisión a hacer su trabajo. Y debido a que estas propuestas habrán nacido del análisis contextualizado de la información científica por un amplio y multidisciplinarlo grupo de profesionales independientes (incluyendo no solo salubristas, epidemiólogos y clínicos, sino también, por ejemplo, economistas, sociólogos, antropólogos, entre otros), las políticas o programas generados desde ellas tendrán mucha mayor probabilidad de ser costo-efectivas ${ }^{(3)}$.

En el Perú, la producción en investigación en salud aún es poca ${ }^{(4)}$. De la poca que se logra hacer, solo la minoría llega a convertirse en intervenciones, programas o políticas de salud. Existe mucha investigación que aún espera ser traducida en acciones específicas en beneficio de la sociedad peruana, por ejemplo, respecto a accidentes de tránsito, neumonías, problemas de alcohol, prematuridad, y depresión (por mencionar únicamente el caso de los cinco problemas de salud que más carga le generan a la sociedad peruana) ${ }^{(5)}$. La investigación en estos, como en otros temas, está en su mayor parte dispersa, perdida, innecesariamente duplicada o conocida por unos pocos. Mientras tanto, muchas de las acciones en las poblaciones de estas áreas, como en otras también, siguen dándose con información parcial, descontextualizada, algunas veces sesgada, o siguiendo idiosincrasias de tomadores de decisiones (6). Esto, naturalmente, lleva a la implementación de acciones poco costo-efectivas y a la descontinuación de otras que sí funcionan.

Recientemente, se ha retomado en nuestro país un interés por fomentar la investigación en salud. Esta iniciativa, liderada por el Instituto Nacional de Salud a través de la 
implementación del Sistema Nacional de Investigación en Salud, tiene el mérito adicional de que trata de orientar los esfuerzos hacia la investigación en prioridades nacionales de salud. Pero, aumentar la producción en investigación, aunque necesario, es solamente avanzar la mitad del camino. Esa iniciativa debe ser complementada con una que traduzca paralelamente los resultados de esas investigaciones en acciones específicas. Esto último es la función que propongo para lo que podríamos llamar el Centro de Gestión para un Desarrollo Sostenido y Coordinado de la Salud (CGDS).

Específicamente, este Centro de Gestión tendría entre sus funciones: a) identificar y organizar la investigación, tanto local como internacional, y traducirla en información contextualizada y contrastada con nuestra realidad, lista a ser usada en el diseño de acciones para el beneficio de poblaciones locales; b) proponer y promover activamente líneas de investigación enfocadas en las brechas de conocimiento que se vayan identificando como urgentes, dadas las necesidades locales; c) identificar y organizar a los actores e instituciones dedicadas a la investigación, y propiciar su interacción con los potenciales usuarios de la investigación (p.ej., tomadores de decisiones); d) identificar, organizar y hacer accesible la investigación producida localmente; e) identificar, organizar y hacer visible información acerca de oportunidades de entrenamiento, financiación, cursos, actividades sociales y otros actos locales e internacionales que puedan ser de interés para la comunidad científica y tomadores de decisiones a nivel nacional; f) organizar y procesar información que deba ser difundida a la población a través de un trabajo cercano con los medios masivos de comunicación; g) establecer trabajo colaborativo de compartir y procesar información con otras instituciones gubernamentales (p.ej., otros ministerios, institutos nacionales, hospitales), universidades, y otras instituciones privadas y extranjeras.

En resumen, se propone crear un centro gravitacional de inteligencia que tenga como actividad principal y cotidiana -y no solo en respuesta a peticiones específicas y eventuales- identificar y reunir información científica, principalmente la producida localmente, para procesarla y traducirla en acciones de salud pública o clínica, fortaleciendo así un círculo virtuoso de investigacióntraducción-investigación en nuestro sistema de salud. Se prevé que una iniciativa como esta tenga impacto a varios niveles, como por ejemplo: a) la generación de acciones en salud costo-efectivas; b) la producción de iniciativas independientes de idiosincrasias individuales o de presiones políticas, que suelen darse en los procesos de toma de decisiones, para asegurar su sostenibilidad; c) facilitar la rendición de cuentas de los tomadores de decisiones; d) fomentar la interacción de actores y el trabajo colaborativo ordenado; e) disminuir la duplicación innecesaria de iniciativas; f) la iniciación de un círculo virtuoso que crea demanda de evidencia científica que estimulará una competencia sana entre las instituciones académicas y de investigación locales.

Finalmente, el Centro, al acelerar los procesos de aplicación de la ciencia en la identificación y control de problemas urgentes, inevitablemente nos conducirá a efectuar innovaciones, a pesar de los escasos recursos. Ello nos llevará a crear liderazgo en Latinoamérica, y quizá en el mundo, respecto a la implementación de un sistema de salud pública que se basa en la evidencia, que constantemente se renueva, y que se da en un contexto donde los recursos son escasos; pero esta vez, serán recursos para ser usados con sabiduría.

\section{Conflicto de Interés}

El autor ha hecho consultorías al Instituto Nacional de Salud del Perú.

\section{REFERENCIAS BIBLIOGRÁFICAS}

1. Lee J. Somewhere, something incredible is waiting to be known. Washington DC: White House Blogs; 2010. [Fecha de acceso: 30 de agosto de 2010]. Disponible en: http://www.whitehouse.gov/blog/Somewhere_something incredible_is_waiting_to_be_known/.

2. Kennedy A, IJsselmuiden C. Building \& strengthening national health research systems. A manager's guide to developing and managing effective health research systems. Geneva: Council on Health Research for Development; 2006.

3. IJsselmuiden C, Matlin SA. Why research for health? Research for health: policy briefings (series) vol. 1. Geneva: Council on Health Research for Development and Global Forum for Health Research; 2006.

4. Alger J, Becerra-Posada F, Kennedy A, Martinelli E, Cuervo LG, Grupo Colaborativo de la Primera Conferencia Latinoamericana de investigación e Innovación para la Salud. Sistemas nacionales de investigación para la salud en América Latina: una revisión de 14 países. Rev Panam Salud Publica. 2009; 26(5): 447-57.

5. VelásquezA. La carga de enfermedad y lesiones en el Peru y las otras prioridades del plan esencial de aseguramiento universal. Rev Peru Med Exp Salud Publica. 2009;26(2):222-31.

6. Fiestas F, Gallo C, Poletti G, Bustamante I, Alarcón RD, Mari, JJ, et al. Improving mental and neurological health research in Latin America: a qualitative study. BMC Public Health. 2009;9:334.

Correspondencia: Fabián Fiestas, $M D, P h D(c)$

Dirección: Av. Honorio Delgado 430, San Martín de Porres, Lima, Perú.

Teléfono: (+511) 985-765-743

Correo electrónico: ffiestas@epi.msu.edu 